\title{
Distribution Line Equipment and Defect Identification Based on Deep Learning
}

\author{
Gege Chen ${ }^{1, *}$ \\ ${ }^{1}$ State Grid Jiangxi Maintenance Company, Nanchang 330029, China
}

\begin{abstract}
In this study, UAVs were used to collect data of distribution line resources, and defects in distribution line equipment and construction process were identified through deep learning. Different algorithms are used to identify the defects of distribution line equipment and construction process. The research will ultimately support regional synchronization and online development for intelligent automatic acceptance of the distribution wire UAV.
\end{abstract}

\section{INTRODUCTION}

The construction quality of distribution lines is an important factor to ensure the safe and stable operation of the power grid. For a long time, spot checks and manual methods have been used for the acceptance of distribution lines. The following problems exist: (1) The manual acceptance is not comprehensive enough. Manual acceptance can only check ground pole problems, which affect the safe and stable operation of distribution lines such as line fittings defects, line loose strands, etc., especially problems above the cross arm, which are difficult to find; (2) Manual acceptance is inefficient. Through investigation, it is found that relying on manual acceptance of the distribution network project throughout the year will take more than half a year of uninterrupted acceptance, which is time-consuming and labor-intensive. (3) Narrow coverage. Manual acceptance cannot touch the acceptance of distribution lines in special areas such as fish ponds, lakes, mountains, hills, resulting in the inability to guarantee the quality of distribution lines in this area; The quality of archived data is not high. Due to changes in the initial design and completion of the project, the as-built drawings and engineering quantities are changed, relying on manual filing is heavy and errorprone.

In recent years, with the rapid development and wide application of digital camera and computer vision technology, it has become possible to intelligently identify electrical equipment and defects by using unmanned aerial vehicle (UAV) to carry cameras and other tools. Huang et al. identified the broken and scattered strands of transmission wires with the algorithm based on radial basis probability neural network [1]. Yang et al. took the Angle between adjacent aluminum wires as the characteristic quantity and used machine learning method to identify the scattered strands of wires [2]. The defect samples studied by these scholars are all from transmission lines. But the operating environment of distribution lines and transmission lines are very different. Therefore, further study is needed to determine whether these methods are applicable to distribution lines.

This research adopts UAV distribution line resource data collection [3], automatic identification of distribution network equipment [4] and defect detection in the construction process, forms distribution network line account information, and completes the display of different types of distribution network line distribution maps. The distribution information of line poles and towers are shown visually and intuitively. The effective data support for the construction of an economic, efficient, and practical intelligent distribution network is provided as well.

\section{IMAGE COLLECTION}

The UAV can carry out intelligent receiving operation and accurately collect images of power distribution line equipment. In addition, refined photo data of the tower, physical ID data and basic data of the pylon can be obtained during image collection, and accurate geographic information and attribute information of the pylon can be obtained, which is very important for the completion of wiring GIS modeling work. In a flight mission, the data of the poles and towers, as well as the refined equipment photos, were collected to realize the basic data collection of the path point route GIS modeling, which can provide the necessary data for the modeling support.

\section{IMAGE IDENTIFICATION}

Image recognition technology is based on the main characteristics of the image. Each image has its characteristics. According to the different device characteristics, many photo samples are prepared, the samples are labeled, and the training model is established. 
In this project, Recognize the refined photos collected by humans and machines, and identify the line nameplates, tower types, circuit breakers, isolating switches, main transformers, and other residential equipment contained in the photos, and you can get the tower types and the names of the equipment on the poles.

\section{1. data preparation}

In the collected distribution network pictures, the equipment and defects are marked. The labeling method is: using the upper left corner and the lower right corner, $\left(\mathrm{x}_{\min }, \mathrm{y}_{\min }, \mathrm{x}_{\max }, \mathrm{y}_{\max }\right)$ to label a target. The marked equipment targets are transformers, fuses, and switches. The marked defect targets include missing pins and broken stocks.

\subsection{Power distribution equipment inspection}

Equipment categories include transformers, fuses, knife switches, etc. Since the equipment target is larger than the defect, the one-stage target inspection model can meet the equipment inspection requirements. In the one-stage target detection model, SSD uses more scale feature map sets (models such as YOLO classify and regress on threelayer feature maps, and SSD classify and regress on sixlayer feature maps). For the project, There are obvious differences in the size of transformers, fuses, and knife switches. It is more appropriate to use SSD as a device detection model.

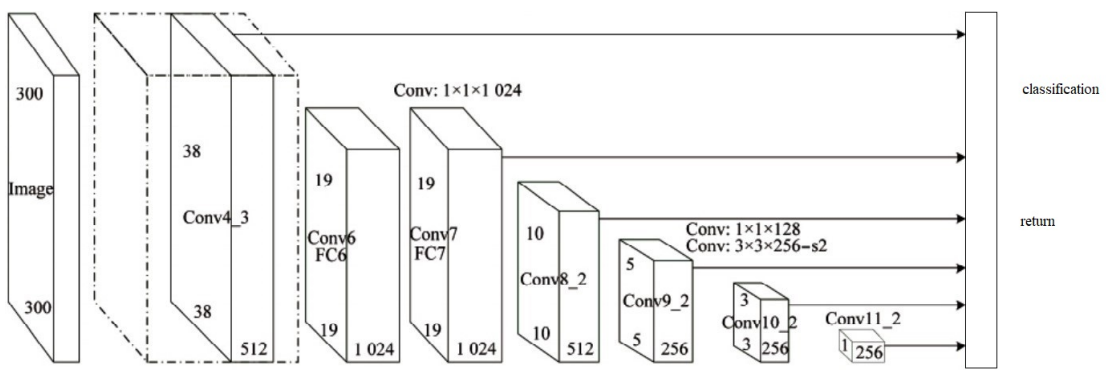

Figure 1 SSD model structure

The feature of $\mathrm{CNN}$ network is that the previous feature map size is relatively large, and the feature map will gradually become smaller through convolution and pooling operation, while the structure of SSD takes advantage of this feature. Both the smaller feature map and the larger feature map are used for detection and output, which is an end-to-end algorithm that directly predicts the target and outputs the bounding box. SSD target detection algorithm is at the leading level in terms of accuracy and efficiency, and the idea that SSD algorithm detects targets of different sizes on feature graphs of different convolution scales by generating candidate boxes makes it have good robustness in various application scenarios and good performance in other target detection problems. So SSD algorithm is feasible to solve the problem of distribution line equipment detection.

The original feature extraction network of Faster Rcnn is VGG16, which is used to extract features from the input image. In the SSD target detection algorithm, all convolutional layers of the VGG network are retained, and the original fully connected layer of the VGG network is replaced with a $3 \times 3$ convolutional layer, and the number of output channels remains unchanged. After the feature extraction network, additional layers are added for multi-scale feature map output. The two-layer feature map output by conv4_3 and fc 7 in VGG and the fourlayer feature map output by the additional layer together form the six-layer multi-scale feature map of the SSD. Finally, the multi-scale feature map is pulled into one dimension and then classified and regression prediction is performed.

The core design concept of SSD [5] algorithm can be summarized as the following points.
The classification of the SSD to remove all connection layer network as a front-end feature extraction, behind its added multi-scale characteristic detection network, by pooling operation will be the size of the layer gradually narrowed, for the size of the same prior box, the characteristics of high-level diagram has high-level semantic information, due to large corresponding receptive field, facilitate testing big goals, However, the feature map at the lower level has detailed information, and the receptive field corresponding to the same range is smaller, which is convenient for detecting small targets. SSD proposes to carry out detection on multiple scales, and the convolution model used for prediction detection at each feature layer is different, which can improve the accuracy of recognition.

Different from YOLO [6], which conducts detection after the full connection layer is adopted, SSD is a full convolutional neural network that directly detects feature images through convolution. For 6 specific convolutional layer outputs in the network, two sets of $3 \times 3$ convolutional cores are used for classification and bounding box regression respectively. The essence of this method is to classify and regression the actual effective receptive fields corresponding to the six feature maps.

SSD borrowed the idea of Faster RCNN and set a different size of default box for each feature graph unit [7].

$$
s_{k}=s_{\min }+\frac{s_{\text {max }}-s_{\min }}{m-1}(k-1), k \in[1, m]
$$

Where $\mathrm{m}$ is the number of layers of the feature graph; smin represents the minimum proportion of the default box in the feature graph; smax represents the maximum proportion of the default box in the feature graph. 
Set the default box of multiple aspect ratios on the same feature layer. When the aspect ratio is 1 , add a default box that is scaled to a so that there are 6 default boxes for each feature graph position. At the same time set the center coordinates of each box as $\left(\frac{c+0.5}{\left|\omega_{k}\right|} \frac{f+0.5}{\left|\omega_{k}\right|}\right)$. Where $\omega_{k}$ is the size of the $k$-th feature map, and $c, f \in\left[0,\left|\omega_{k}\right|\right)$. Then intercept the default frame coordinates so that they are always within $[0,1]$.

The distribution network is not only in the wild but also in the streets and other places. As shown in the picture sample of the distribution network equipment, there is a lot of vegetation, bridges, buildings, etc. in the background of the picture. And due to the influence of illumination, shooting angle, etc., identification is difficult.
To improve the feature extraction capability of the model, the original feature extraction structure VGG16 of the SSD model is replaced with ResNet50. Compared with the VGG16 network, the Resnet50 network has a deeper network structure and a stronger feature description capability. Because scholars generally believe that improving the capacity of neural network can make the network approach the optimal solution function, and the computing power of computer is constantly improving, deeper neural network is constantly proposed. As the network deepens, some problems emerge that do not exist in the shallow network. The researchers found that simply piling up network layers was detrimental to the deep web.RES Net can be applied to the deep neural network structure, so that the accuracy of the neural network with a large increase in depth does not decrease.

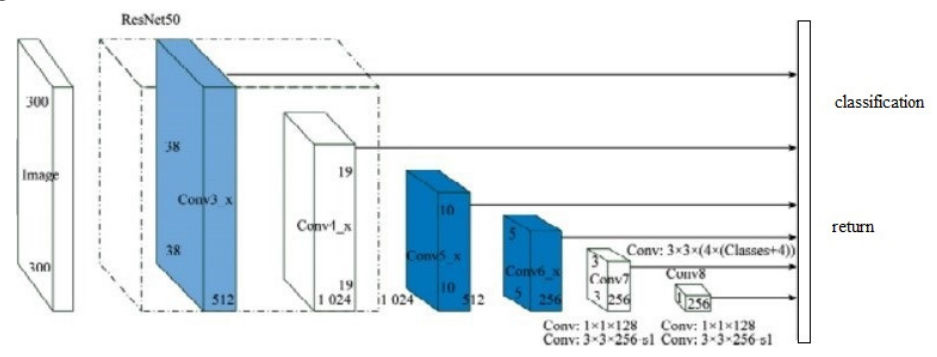

Figure 2 replaced ResNet50-SSD model

In the network structure, the jump connection of the feedforward neural network is used. At the same time, the $1 \times 1$ volume is used to reduce the number of parameters and improve the operation efficiency. When the dimension of the jump connection is not matched, the same dimension is reflected by the $1 \times 1$ convolution and then the phase is added. The convolution residual unit is shown in (2).

$$
y=F\left(x, W_{i}\right)+W_{j} x
$$

Where $x$ and $y$ represent input and output of the layer respectively. $F\left(x, W_{i}\right)$ is the residual mapping that we learned. Wi table shows dimensional scalar enantiomorphic, when $\mathrm{Wj}$ is a unit vector, a $1 \times 1$ convolution is required to match the dimensions.

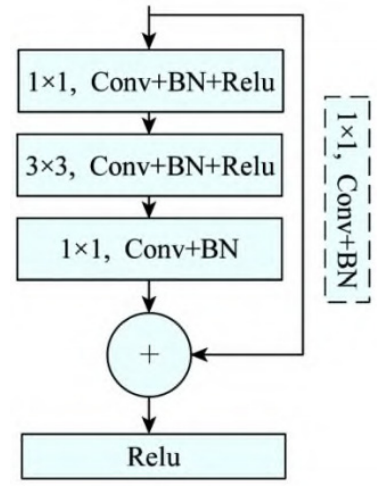

Figure 3 Residual structure unit

The loss of the distribution network equipment detection model consists of two parts, one is the classification loss, and the other is the regression loss. The classification loss is calculated using Focal loss, and the regression loss is smooth_11 loss. When the SSD model classifies, it directly classifies all detection frames, and the background class accounts for the vast majority, resulting in a serious imbalance between target classes. Therefore, Focal loss is used for classification loss which is given as follows:

$$
F_{\text {loss }}(y)=-\alpha_{y} \log \left(p_{y}\right)\left(1-p_{y}\right)^{\gamma}
$$

By adjusting the ay parameter in focal loss, the weight of each category in the data set can be balanced. By adjusting the $\gamma$ parameter in focal loss, the weight of easyto-divide samples in the sample can be reduced. Using focal loss can effectively alleviate the impact of sample target imbalance during the training process and improve the detection accuracy.

\subsection{Construction process defect detection}

Defect categories include missing pins, broken stocks, etc. Due to the smaller defect targets, the two-stage target detection algorithm has better detection accuracy for smaller targets. The project uses the faster R-CNN target detection algorithm for the distribution network defects. Faster R-CNN model structure:

Due to the small size of the defect target, the captured distribution network defect pictures can only be well identified with a larger resolution. During training, maintaining the resolution of the original image requires a large amount of memory and video memory. To reduce the memory usage, the feature extraction network VGG in 
Faster Rcnn is now replaced with the Inception v2 network.

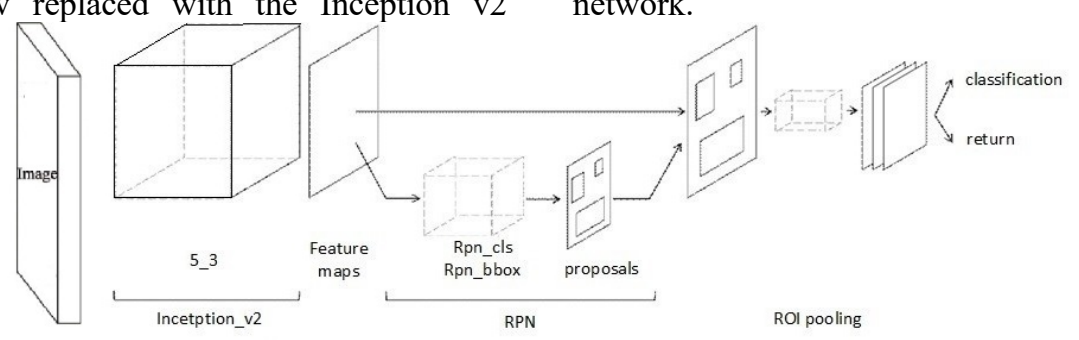

Figure 4 Faster R-CNN model structure

Inception-v2[8], modified to the version in February 2015 , reduces the TOP-5 error rate of ILSVRC to $4.8 \%$. We will use this algorithm to extract the convolution features of construction process defects in the implementation. Inception v2 adds the Batch Normalization, so that each neuron input is distributed to the standard normal distribution with mean 0 and variance 1. This relieves the problems of neural network training difficulty and slow convergence speed. At the same time, two consecutive $3 \times 3$ convolutional layers are used to replace the $5 \times 5$ convolutional layer, thus reducing the number of parameters without reducing the expressive power.

Compared with the VGG network, the Inception network requires less memory under the same conditions, and the calculation speed is faster. The Faster R-CNN target detection algorithm is a two-stage model. In the first stage, RPN judges the background scene and generates a region of interest suggestion. The second stage is to classify and regress the features of the region of interest generated by ROI pooling.

\section{DISTRIBUTION NETWORK MODELING}

The construction of the distribution line ledger is the basis for the diagrammatic expression of lines. According to the tower information, space coordinate data of the tower is displayed at the corresponding position on the map, and the line name, tower name, equipment name, etc. of the identified tower are established according to the image recognition technology to establish a unique association Relationships, form standard data that meets the line drawing, record the data information related to the line tower equipment, and complete the construction of the distribution line ledger.

1) Spatial data processing: The tower coordinate information collected by the drone flight is processed by data processing, attribute assignment, and other operations, and the position of the line tower is accurately located on the map. According to the position of the tower and related attribute information, the line direction connection is automatically realized, and the location of the line tower is located. Towards and other information.

(2) Image recognition equipment information: The image recognition technology is utilized to perform image recognition on the refined tower photos of the distribution network, complete the identification of the tower line, tower number, tower physical ID, equipment on the tower column, and other information, and construct lines and towers based on main information such as line name and tower number. The associated relationship with its equipment, record complete tower attribute information.

\section{ASSET VERIFICATION}

Asset verification realizes inventory verification of distribution network line equipment assets and identification verification of construction process defects.

\subsection{Equipment asset verification}

Equipment asset verification, based on the tower equipment information identified from the refined photos and images, construct a line ledger. The ledger records line name, line tower number, and main equipment information connected to the tower to form a field verification Tower equipment data ledger.

According to the coding rules, the detailed asset equipment system to which the equipment belongs can be confirmed from the two identification codes in the code, and all the RFID codes contained in the current tower are analyzed, and the corresponding equipment information is retrieved from the detailed asset equipment system to form equipment information that the base tower should have in the equipment account.

\subsection{Construction process verification}

Construction process verification is a verification analysis of the defects of the line poles and towers. On the basis of defect identification, the photo information can be further extracted, and the defect type of each basic rod and tower can be accurately obtained. This can assist users to verify the defects of poles and towers in the construction process, and eliminate the defects in time, so as to ensure the safe operation of the line.

\subsection{Connect with grid GIS}

UAV data interaction mainly uses database synchronization technology. To achieve data synchronization, it is necessary to construct the result data structure of the receiving intelligent acceptance system in the Jiangxi intelligent power supply service command platform database. On this basis, push the data to the API interface provided by the power supply service command platform, store the data in the result data structure, and then synchronize the data with the grid GIS system through the data synchronization background program. 


\section{CONCLUSION}

UAVs are used to collect defects pictures of power distribution line equipment and construction process, and deep learning is used to realize the identification of equipment and defects, which is helpful to improve the utilization rate of power distribution line engineering acceptance and construction, further strengthen the accurate research and judgment of equipment defect identification, and improve the safe operation level of power grid.

\section{REFERENCES}

1. Huang Xinbo, Zhang Xiaoling, Zhang Ye. "State Identification of Transmission Line Defect Based on Radial Basis Probabilistic Neural Network." Automation of Electric Power Systems. vol. 44,2020.

2. Yang Zhiyong, Wang Wei, Su Fan. "Research on Untwisted Strand of Overhead Transmission Lines Based on Digital Image Processing Method." Electric Power. vol. 47, 2014

3. Chen, Binghuang, and X. Miao. "Distribution Line Pole Detection and Counting Based on YOLO Using UAV Inspection Line Video." Journal of Electrical Engineering and Technology, vol. 3, 2019.

4. Fengao, Zhang, and L. I. Guoping. "Automatic Identification System of Low-voltage Distribution Network Equipment Based on Carrier Communication Technology." Zhejiang Electric Power, 2017.

5. Su Juan, Yang Long, Huang Hua. "Improved SSD algorithm for small-sized SAR ship detection." Systems Engineering and Electronics. vol. 42, 2020.

6. QI Jie, JIAO Liangbao, " Bird Nest Detection on Transmission Tower Based on Improved SSD Algorithm." Computer Systems \& Applications, vol. 29, 2020.

7. Li Weixing, Zheng Wulue, Wang NingZhao, Hanghang, "Research on Detection Method of Insulator Defects on Transmission Lines Based on SSD Algorithm." Instrumentation Customer, vol. 26, 2019.

8. Ioffe S, Szegedy C. Batch normalization "Accelerating deep network training by reducing internal covariate shift. " In Proceedings of The 32nd International Conference on Machine Learning. Vol. 1,2015 